\title{
BMJ Open Rationale and design of BERLIN VT study: a multicenter randomised trial comparing preventive versus deferred ablation of ventricular tachycardia
}

\author{
Roland Richard Tilz, ${ }^{\oplus}$ Karl-Heinz Kuck, ${ }^{2}$ Stephan Kääb, ${ }^{3}$ Karl Wegscheider, ${ }^{4}$ \\ Annette Thiem, ${ }^{5}$ Beate Wenzel, ${ }^{5}$ Stephan Willems, ${ }^{6}$ Daniel Steven ${ }^{7}$
}

To cite: Tilz RR, Kuck K-H, Kääb S, et al. Rationale and design of BERLIN VT study: a multicenter randomised trial comparing preventive versus deferred ablation of ventricular tachycardia. BMJ Open 2019;9:e022910. doi:10.1136/ bmjopen-2018-022910

- Prepublication history for this paper is available online. To view these files, please visit the journal online (http://dx.doi. org/10.1136/bmjopen-2018022910).

Received 20 April 2018 Revised 10 January 2019 Accepted 12 February 2019
Check for updates

(C) Author(s) (or their employer(s)) 2019. Re-use permitted under CC BY-NC. No commercial re-use. See rights and permissions. Published by BMJ.

For numbered affiliations see end of article.

Correspondence to

Professor Roland Richard Tilz; tilz6@hotmail.com

\section{ABSTRACT}

Introduction Catheter ablation (CA) has shown to effectively reduce the burden of ventricular tachycardia in patients with implanted cardioverter-defibrillator (ICD). However, in patients with ICD implantation for secondary prevention of ventricular tachycardia (VT), the appropriate time point of CA and its effect on mortality and heart failure progression remains a matter of debate. Methods and analysis We present the design of the ongoing preventive $a \underline{B}$ lation of $v \underline{E}$ ntriculartachycaRdia in patients with myocardiaLINfarction (BERLIN VT) study that aims to prospectively enrol 208 patients with a stable ischaemic cardiomyopathy, a left ventricular ejection fraction of $30 \%$ to $50 \%$ and documented ventricular tachycardia. Patients will be 1:1 randomised to undergo CA at the time of ICD implantation or CA after the third appropriate ICD shock for ventricular tachycardia. ICD implantation will be performed in all patients. The primary endpoint is defined as the time to first event comprising all-cause mortality and unplanned hospital admission for congestive heart failure or for symptomatic VT/ ventricular fibrillation. The patients will be followed until study termination according to the event driven design. Completion of enrolment is expected for mid of 2019. Ethics and dissemination The study had been approved by the "Ethik-kommission der Landesärztekammer Hamburg" as well as the local institutional review boards for each of the participation sites. The results of the trial will be published in peer-reviewed journals Trial registration number NCT02501005.

\section{INTRODUCTION}

The therapy of choice to prevent sudden cardiac death in survivors of myocardial infarction presenting with recurrent ventricular tachycardia (VT) is the implantation of an implantable cardioverter-defibrillator (ICD). ${ }^{1-5}$ The ICD treatment, however, does not cure or prevent VT, but merely terminates VT episodes by antitachycardia pacing or shocks. Whether adequate or inadequate, ICD shocks are often painful, associated with a lower quality of life, clinically significant depression and anxiety, deterioration of
Strengths and limitations of this study

- This is a prospective, randomised, controlled, open and multicentre trial involving 30 European centres therefore allowing for generalisability of the data.

- Telemonitoring will be performed in all patients to allow for a continuous rhythm monitoring and a very close follow-up.

- The data that will result from this study cannot be transferred to patient with non-ischaemic cardiomyopathy because only patients with ischaemic cardiomyopathy and ventricular tachycardia will be enrolled.

- Due to the limited patients number eligible for VT trials, this study is not powered to demonstrate a mortality benefit and no such trial will be conducted in the next decade as it would require thousands of patients to demonstrate an impact on mortality.

heart failure and an increased risk of all-cause mortality. ${ }^{6-8}$

In post myocardial infarction patients, VT typically originates from a relatively discrete region of left ventricular myocardium within or bordering the infarct zone. ${ }^{910}$ Several therapeutic approaches, such as surgical excision, pharmacological suppression and catheter ablation, are insufficient to prevent sudden cardiac death. ${ }^{11-14}$ Antiarrhythmic drugs have little or no impact on mortality or ICD shock reduction. ${ }^{14}$ Transfemoral catheter-based ablation procedures are increasingly performed to treat recurrent VTs. ${ }^{15}$ Ablation strategies include linear ablation traversing the border zones of infarcted and normal tissue or anatomical boundaries, elimination of all late potentials, fractionated potentials and local abnormal ventricular activity or isolation of the endocardial substrate. ${ }^{15-20}$ Specific features of electrograms denoting the site of origin of the VT and its response to certain pacing manoeuvres have been described. ${ }^{21-23}$ 
In the last decade, catheter ablation has evolved from an almost experimental procedure to a standard therapy for scar-related VTs. Recent studies have evaluated the efficacy of preventive catheter ablation before ICD implantation in patients with VT and ischaemic cardiomyopathy. There was a non-significant trend toward a lower mortality following VT ablation. ${ }^{24}$ Two retrospective single-centre analyses indicated that patients may benefit from an early ablation approach more than from ablation performed after several ICD shocks. ${ }^{25} 26$

\section{METHODS AND ANALYSIS \\ Primary hypothesis}

The primary hypothesis is that, in chronic post-infarction patients with recurrent VT, preventive VT ablation prior to ICD implantation improves the primary endpoint compared with the ablation after the third appropriate ICD shock.

\section{Primary objective}

The primary objective of the ongoing preventive a $\underline{B}$ lation of $\underline{\underline{E}}$ ntricular tachyca $\underline{R}$ dia in patients with myocardia $\underline{L}-$ INfarction (BERLIN VT) study is to investigate whether a strategy to ablate a VT prior to ICD implantation is superior to the established ablation strategy after the third ICD shock triggered by VT. We hypothesised that the preventive ablation would not reduce only the number of shocks but also mortality and morbidity.

\section{Secondary objective}

Secondary objectives are: (1) to observe the rate and occurrence of ventricular arrhythmias, related events and the resulting interventions triggered by the ICD, (2) to assess the rate and occurrence of cardiac morbidity and mortality and (3) to determine accumulated changes in quality of life during the first 12 months.

\section{Study design}

The BERLIN VT study is a prospective, randomised, controlled, open and multicenter trial. Institutional review board approval was obtained at each of the participating centres. About 30 European centres will participate in the study. Study sites are selected based on the availability of an experienced electrophysiologist to perform the ablation procedure. It is required that the electrophysiologist has performed at least 50 catheter ablations and $10 \mathrm{VT}$ ablations in patients with ischaemic or non-ischaemic cardiomyopathy within the last year. There are no restrictions for patient numbers per site.

Patients will be randomised in a 1:1 matrix. To minimise any bias due to uneven distribution of patients with congestive heart failure or atrial fibrillation between the two groups, the randomisation is stratified by type of implanted ICD (with or without resynchronisation therapy) and by history of atrial fibrillation.

\section{Participants}

The study population will consist of patients with an indication for an ICD as a means of secondary prevention after documented sustained VT in the absence of a reversible cause, but with concomitant coronary artery disease, previous myocardial infarction and moderately reduced left ventricular ejection fraction (30\% to $50 \%)$ estimated by cardiac MRI, three-dimensional (3D) echocardiography or ventriculography. Patients with a markedly reduced ejection fraction $(<30 \%)$ will not be included after the Ventricular Tachycardia Ablation in Coronary Heart Disease (VTACH) study has shown that preventive VT ablation in these patients does not reduce VT/ventricular fibrillation (VF) recurrence. A detailed list of inclusion and exclusion criteria is provided in table 1 .

A signed informed consent form will be obtained from every patient prior to any trial-related procedure. The consent form has been approved by the corresponding ethics committee or institutional review board, and has been written in accordance with the country-specific applicable data privacy acts, the Declaration of Helsinki and the applicable laws for research using medical devices.

\section{ICD implantation and programming}

In the treatment group, ICD will be implanted within 2 weeks of VT ablation, desirably during the same hospital stay, and in the control group, ICD will be implanted within 2 weeks after enrollment. The implantation will be conducted according to standard surgical technique. The lead system will be transvenous. In case of a system upgrade from pre-existing pacemaker to ICD, the management of pre-existing leads is left to the discretion of the investigator. Also defibrillation threshold testing will be performed at the discretion of the investigator.

All implanted ICD devices will be manufactured by the study sponsor in order to achieve a uniform process of data collection and processing and to warrant Home Monitoring function. Home Monitoring will be activated in both treatment groups. The treating physician will have full access to the Home Monitoring data. Use of Home Monitoring is aimed at assuring a timely recognition of ICD therapies and other actionable clinical events.

Uniform settings are recommended for Home Monitoring alerts and for ICD parameters of arrhythmia detection and therapy (table 2). Care will be taken to prevent unnecessary and inappropriate shocks, for example, by long detection windows.

\section{Catheter mapping and ablation}

Prior to the ablation procedure, transthoracic or transoesophageal echocardiography is required at least in patients with atrial fibrillation to exclude the presence of an intracardiac thrombus. If a thrombus is present, adequate measures will be taken to dissolve it and the VT ablation will be postponed.

A two-step catheter ablation procedure will be performed within 2 weeks of enrollment in the preventive ablation arm (the treatment group).

In a first step, a diagnostic electrophysiological study will be done to induce the clinical VT. Specifically, the right ventricle will be stimulated at two sites with up to three 
Table 1 Inclusion and exclusion criteria

\section{Inclusion criteria}

1. History of remote myocardial infarction

2. Left ventricular ejection fraction $\geq 30 \%$ to $\leq 50 \%$ as estimated by cardiac MRI, 3D echocardiography or via ventriculography within 30 days before enrollment

3. Documentation of sustained VT by any kind of ECG including 12-lead ECG, Holter ECG, rhythm strip, event monitoring, event recorder or pacemaker within 30 days before enrollment

4. ICD indication for secondary prevention

5. Patients who are planned to be implanted with Biotronik ICDs (single-chamber, dual-chamber, triple-chamber or DX device)

6. Patient has provided written informed consent

7. Patient accepts activation of Home Monitoring

\section{Exclusion criteria}

1. Age $<18$ years or $>80$ years

2. Known arterial or venous thrombosis

3. Class IV NYHA heart failure

4. Valvular heart disease or mechanical heart valve precluding access to the left ventricle

5. Acute myocardial reinfarction or acute coronary syndrome

6. Cardiac surgery involving cardiotomy within the past 2 months

7. Patients requiring chronic renal dialysis

8. Thrombocytopenia or coagulopathy

9. Incessant VT or electrical storm

10. Bundle-branch reentry tachycardia as the presenting VT

11. Pre-existing ICD

12. Pregnancy or breast feeding women

13. Acute illness or active systemic infection

14. Other disease process likely to limit survival to $<12$ months

15. Significant medical problem that in the opinion of the principal investigator would preclude enrollment in the study

16. Unwillingness to participate or lack of availability for follow-up

17. Participation in another interventional clinical investigation during the course of the study (participation in a noninterventional clinical investigation is allowed)

DX, ICD with a single lead allowing atrial sensing via floating dipole; ICD, implantable cardioverter-defibrillator; MRI, magnetic resonance imaging; NYHA, New York Heart Association; VT, ventriculartachycardia; 3D; three-dimensional.

extrastimuli at two drive cycle lengths, until a coupling interval of at least $220 \mathrm{~ms}$ is reached between the drive train and the extrastimuli or until a sustained $(30 \mathrm{~s})$ VT is induced. To be considered as a clinical arrhythmia, an induced VT must match the documented clinical VT

Table 2 Implantablecardioverter-defibrillator settings

\begin{tabular}{|c|c|}
\hline Parameter & Setting \\
\hline \multicolumn{2}{|l|}{ Mandatory ICD settings } \\
\hline Home Monitoring & ON \\
\hline \multicolumn{2}{|l|}{ Recommended ICD settings } \\
\hline \multicolumn{2}{|l|}{ VF zone } \\
\hline $\begin{array}{l}\text { Cut-off cycle length } \\
\text { Detection counter } \\
\text { Tachycardia therapy }\end{array}$ & $\begin{array}{l}270-300 \mathrm{~ms} \\
18 \text { out of } 24 \text { or higher } \\
\text { ATP one shot and shock } \\
\text { therapy }\end{array}$ \\
\hline \multicolumn{2}{|l|}{ VT zone* } \\
\hline $\begin{array}{l}\text { Cut-off cycle length } \\
\text { Detection counter } \\
\text { Tachycardia therapy } \\
\text { SMART detection† } \\
\text { ATP optimisation }\end{array}$ & $\begin{array}{l}60 \mathrm{~ms} \text { above slowest VT } \\
\text { on decision of investigator, } \\
\text { as high as possible } \\
\geq 3 \text { ATPs and shock therapy } \\
\text { ON } \\
\text { ON }\end{array}$ \\
\hline
\end{tabular}

*It is recommended to programme at least one VT zone. †Or MorphMatch.

ATP, antitachycardia pacing; ICD, implantable cardioverterdefibrillator; VF, ventricular fibrillation; VT, ventricular tachycardia. in morphology and rate $( \pm 20 \mathrm{bpm})$. Ischaemic patients with systolic left ventricular dysfunction and an episode of spontaneous sustained VT will mostly have more than one VT morphology inducible. Since any inducible VT can potentially become a clinical VT, an attempt will be made to ablate all inducible morphologies and not only the clinical VT. ${ }^{27}$ If no VT is inducible, solely a substratebased ablation approach will be used.

In the second step, a 3D electroanatomical reconstruction of the left ventricle will be performed. For mapping, a $3.5 \mathrm{~mm}$ irrigated-tip ablation catheter or a multielectrode mapping catheter will be used. Bipolar endocardial voltage of $\geq 1.5 \mathrm{mV}$ will be defined as 'normal'. Late potentials will be defined as any low voltage $(<1.5 \mathrm{mV})$ electrogram with a single component or with multiple continuous delayed components separated from the higher amplitude component of the local ventricular electrogram by $\geq 20 \mathrm{~ms}$ and recorded after the end of the surface QRS. ${ }^{20} 28$

After completion of the 3D electroanatomical map, catheter ablation using irrigated radiofrequency energy (recommended maximum power: 30 to 50 watts) will be performed within the entire area displaying late potentials until all late potentials get eliminated or until 1 hour of cumulative radiofrequency energy delivery is reached without late potential elimination. The acute ablation success is defined as the elimination of all late potentials and the absence of any inducible VT. 


\section{Primary endpoint}

The primary endpoint is defined as the time to first event comprising all-cause mortality and unplanned hospital admission for congestive heart failure or for symptomatic VT/VF, which will be determined by an independent endpoint assessment committee. Hospitalisation is defined as admission to a hospital involving at least one overnight stay. Hospitalisation for heart failure includes admission for first diagnosis of heart failure as well as worsening of heart failure events. Daycare admission for the acute treatment of heart failure is included in the primary endpoint if heart failure was treated acutely, for example, with intra-venous or oral medication for heart failure including diuretics, vasodilators or inotropic agents.

\section{Secondary endpoints}

Secondary endpoints of the study will assess patient care parameters: time to first sustained VT/VF, time to first appropriate ICD therapy, time to first inappropriate ICD therapy, time to all-cause mortality, time to cardiac mortality, time to first unplanned all-cause hospitalisation, time to first unplanned cardiac hospitalisation and change in quality of life from enrollment to the 12 month follow-up based on the short form (SF)-36 physical and/ or mental component score.

\section{Safety analysis}

Procedural complications will be collected and analysed regarding the frequency and type of complications. Moreover, all adverse events will be reported according to regulatory requirements and monitored by a Data Safety Monitoring Board. This board will review accumulating study data to address patient safety and ethical issues of the study. Based on the interim data, the board will give a recommendation to the sponsor whether to continue the clinical investigation as planned, to modify the study protocol or to stop the clinical investigation for futility.

\section{Follow-Up}

Clinical visits are scheduled for 3 and 6 months after the index ablation and every 6 months thereafter, until collection of sufficient endpoint data (figure 1). The follow-up evaluations will consist of a physical examination, ICD interrogation with storage of programmer data, evaluation of VT episodes and SF-36 quality of life assessment. Clinically relevant events during follow-up (eg, syncope, electrical storm, cardiac hospitalisation) will be recorded. Home Monitoring data will be continuously reviewed to recognise conditions requiring medical interventions. It should be considered to call a patient in after receiving any of the preset Home Monitoring event alerts.

\section{Statistical methods}

\section{Sample size}

An O'Brien-Fleming group sequential design has been chosen with three interim analyses for early stopping due to superiority or futility or, otherwise, with one final analysis. The study has a statistical power of $80 \%$ to accept

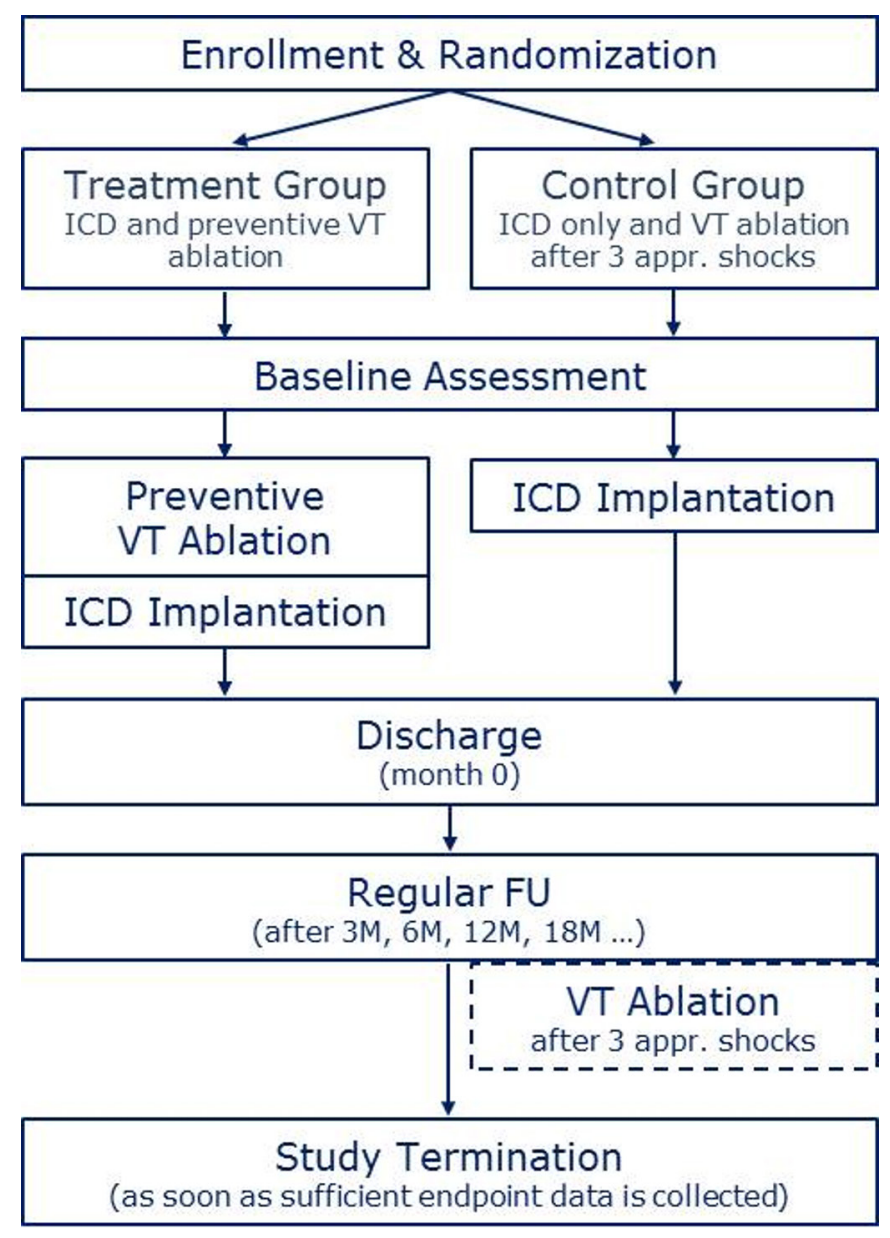

Figure 1 Preventive aBlation of vEntricular tachycaRdia in patients with myocardiaL INfarction study design. Appr., appropriate; FU, follow-up; ICD, implantable cardioverter-defibrillator; M, months; VT, ventricular tachycardia.

the one-sided primary hypothesis (at least after the final analysis) based on a significance level of $2.5 \%$ and given a HR between the treatment and control groups of 0.525 , derived from a retrospective analysis of raw data of the VTACH study and considered to be clinically relevant and worth detecting. Using the SEQDESIGN procedure of SAS 9.4, a total of 85 patients with primary endpoint event are required for the final analysis. If an interim result exceeds or falls below the pre-specified stopping boundaries, the study has to be terminated earlier for superiority or futility.

In the control group of the VTACH study, the cumulative rate of events chosen as primary endpoint for the BERLIN VT study was $61 \%$ in 2 years, which results in a hazard rate of 0.47 per year for exponential survival. However, this event rate seemed too high in the light of the results of more recent studies concerned with the clinical effects of shock prevention (eg, Multicenter Automatic Defibrillator Implantation Trial - Reduce Inappropriate Therapy [MADIT RIT] and Avoid Delivering Therapies for Non-Sustained Arrhythmias in ICD Patients III [ADVANCE-III] trials $^{2930}$ ). Although these trials did not 
directly report the combined primary endpoint assessed in the BERLIN VT study, they delivered surrogates that led to the assumption of a lower hazard rate of the control group of 0.32 per year. Consequently the expected hazard rate in the therapy group is 0.17 per year. Taking into account an exponential loss of $8 \%$ per year due to drop-outs, a 1.5 year enrollment period and 1.5 years of follow-up, 104 patients should be enrolled in each group, or 208 in total, to observe the required endpoints.

\section{Statistical analysis plan}

Details on the projected statistical analyses are described in a statistical analysis plan. All endpoints will be analysed based on the intention to treat principle. The primary endpoint will also be analysed based on a per-protocol population consisting of all patients that were treated according to their randomisation group and according to all requirements set up by the study protocol, including ablation in the control group after three appropriate shocks confirmed by the central shock adjudication board.

The time-to-first-event hypotheses will be analysed via Cox regression analysis using the history of atrial fibrillation and planned cardiac resynchronisation therapy as strata for the primary endpoint. Quality-of-life related hypotheses will be analysed with Wilcoxon testing.

For continuous variables, the mean value, SD and quartiles will be calculated. For nominal or ordinal variables, the absolute and relative frequencies based on non-missing data will be calculated. For two-sided and one-sided statistical tests, a $p$ value of $<5 \%$ and $<2.5 \%$ will be considered statistically significant, respectively. There will be no adjustment for multiplicity for all secondary hypotheses and for further analyses, and all findings will express supportive evidence for the primary hypothesis only. All calculations will be carried out using the SAS 9.4 statistical software or upgrades (SAS Institute Inc., Cary, NC, USA).

\section{Data collection and handling}

All study-relevant patient data will be documented pseudonymously in electronic case report forms (eCRF) using the clinical data management system (CDMS) 'iMedNet' of the vendor MedNet Solutions. Only authorised users with fixed roles have access to the CDMS. Every access is automatically logged and changes of the clinical data are stored in independent audit trails. The CDMS is verified and validated accordingly.

After data entry into the CDMS, the clinical data is automatically checked with programmed quality checks. Additionally, the eCRF will be checked against source data by clinical monitors during periodic monitoring visits as described in a monitoring plan. All further details of data management are described in a clinical data management plan.

\section{Patient and public involvement}

Patients and public were not involved in the design, recruitment and conduct of this study. The results of this study will be made available to the patients and the public by publication in a peer-reviewed journal.

\section{DISCUSSION}

The BERLIN VT Study was designed to evaluate the impact of preventive VT ablation on all-cause mortality and on unplanned hospital admission for congestive heart failure or VT/VF in patients with history of myocardial infarction who would otherwise undergo VT ablation after the third appropriate ICD shock.

\section{Impact of ICD shocks}

Implantation of an ICD in symptomatic patients with ischaemic cardiomyopathy either with impaired left ventricular function ( $<35 \%$, primary prophylaxis) or with hemodynamically unstable VT is a class I indication according to the current guidelines. ${ }^{5}$ There are several trials indicating that primary and secondary prevention reduces the risk of arrhythmogenic death in these patients. ${ }^{3} 14$ 31-33 However, ICD shocks themselves not only are related to pain and anxiety leading to psychological distress but also impact mortality of their recipients irrespective of being adequate or inadequate. ${ }^{6}$ Therefore, it is reasonable to assume that a reduction in ICD shock delivery may impact mortality. A variety of studies in the last years have shown that more conservative ICD programming with longer waiting intervals and less therapies can decrease mortality by reducing ICD shock delivery without increasing the incidence of adverse events such as syncope ${ }^{29} 3034$ Previous studies have also shown that catheter ablation is able to reduce VT recurrence and ICD shocks significantly. ${ }^{35}$ However, no published trial so far showed significant reduction in mortality after catheter ablation, mainly due to the fact that studies focused on the ICD shock recurrence and were underpowered to reveal differences in mortality. ${ }^{24}$

\section{Preventive catheter ablation}

To date, three randomised studies on the impact of 'preventive' ablation in the setting of ischaemic VT have been published. Substrate Mapping and Ablation in Sinus Rhythm to Halt Ventricular Tachycardia (SMASH VT), VTACH, and Substrate Modification Study (SMS) evaluated the impact of preventive catheter ablation on different outcome measures such as time to first recurrence of VT/VF and freedom from any appropriate ICD therapy, ${ }^{24637}$ demonstrating a significant reduction of VT episodes, ICD therapies and ICD shocks in the ablation arm. In two of these studies, the reduction of VT recurrence was around $50 \% .^{24}{ }^{36}$ There are several reasons for the observed outcome differences between the studies, including different study protocols regarding concomitant antiarrhythmic drug therapy and ICD programming algorithms, also leading to marked differences in VT/VF recurrence as detected by the device Holter. ${ }^{37}$ The ideal timing of catheter ablation remains a matter of debate. Retrospective single-centre data indicate that a longer 
interval from shock occurrence to VT ablation is associated with a worse outcome, as opposed to patients undergoing the interventional therapy earlier. ${ }^{25} 26$ Therefore, the BERLIN VT trial evaluates the timing of catheter ablation (early vs after three shocks).

\section{Comparable recent studies}

There are some ongoing studies investigating comparable outcome parameters in patients with structural heart disease requiring ICD interventions. First, the Does Timing of VT Ablation Affect Prognosis in Patients with an ICD (PARTITA) trial aims to recruit 590 patients undergoing catheter ablation at the time of first ICD shock versus after an electrical storm (>3 ICD discharges within 24 hours). In a run-in phase, PARTITA will also evaluate the impact of antitachycardia pacing on ICD shock delivery. Due to the large projected number of patients and the selected endpoints (electrical storm) the study is not expected to end before September 2020 (ClinicalTrials.gov identifier: NCT01547208).

Second, the Pan-Asia United States PrEvention of Sudden Cardiac Death Catheter Ablation Trial (PAUSE-SCD; ClinicalTrial.gov identifier: NCT02848781) aims to recruit patients with arrhythmogenic right ventricular cardiomyopathy (ARVC), dilated cardiomyopathy and ischaemic cardiomyopathy with ejection fraction $<50 \%$ and inducible monomorphic VT at the time of primary or secondary ICD implantation. These patients are considered to be higher risk for recurrent VT and eligible for randomisation. Subjects will be 1:1 randomisation of ICD implantation with optimal medical therapy versus ICD implantation with adjunctive catheter ablation. The study will be completed until February 2023. Furthermore, the Ventricular Tachycardia Antiarrhythmics or AblatioN In Structural Heart Disease 2 (VANISH 2; ClinicalTrials.gov identifier: NCT02830360) aims for comparison of antiarrhythmic drug treatment and catheter ablation as a first line treatment for VT in patients with structural heart disease. The study follows and will complete the results of the VANISH trial that showed inferior efficacy for antiarrhythmic drug escalation versus catheter ablation in patients with recurrent VT. ${ }^{38}$

Several other studies that had been designed to study the effect of preventive VT ablation were interrupted prematurely due to a low enrolment rate: The Indian Trial of Endocardial Ventricular Substrate Ablation to Prevent Recurrent VT Events (INTERVENE; ClinicalTrial.gov identifier: NCT02301390), the Catheter Ablation Versus Amiodarone for Shock Prophylaxis in Defibrillator Patients With Ventricular Tachycardia (CEASE-VT; ClinicalTrial.gov identifier: NCT01097330) and the Substrate Targeted Ablation Using the FlexAbility Ablation Catheter System for the Reduction of Ventricular Tachycardia (STAR VT; ClinicalTrials.gov identifier: NCT02130765) trial.

The data that will be published in the next years will hopefully provide sufficient background information for decision making regarding the point in time of interventional therapy, the type of intervention (substrate vs ablation of the clinical VT), the impact of underlying conditions (ischaemic vs dilated cardiomyopathy) and clinical circumstances such as antitachycardia pacing delivery versus ICD shocks.

\section{Heart failure and telemonitoring}

The patients investigated in the sudden cardiac death and VT/VF studies die not only from arrhythmic death. The underlying disease progression, such as worsening of heart failure, also can play a major role as a cause of death. This aspect has not sufficiently been addressed in previous secondary and primary prevention studies. To focus on this important aspect, the primary combined endpoint in the BERLIN VT study includes not only hospitalisation for symptomatic VT/VFrecurrence but also hospitalisation for congestive heart failure and worsening of heart failure. The design of the study reflects the fact that both study arms should contain optimal medical treatment along with early detection of disease progression by Home Monitoring of the implanted devices. The aim of this particular study design was to warrant a more focused view on the effect of catheter ablation once data analysis is performed and to have less interference with endpoint due to heart failure worsening. The telemonitoring data along with the follow-up and intervention details that will be provided may also help evaluate predisposing factors for arrhythmic events and heart failure exacerbation.

\section{Recruiting VT ablation candidates}

The recruitment of patients for previous VT trials, especially those being performed in Europe, has been slower than anticipated. This is partly due to the inclusion criteria aiming for patients that are relatively rare in clinical practice. The modest recruitment was also due to the referral of patients to the respective specialised centre. For future studies and standard clinical care, VT units should be established in specialised centres in a way to warrant timely care and ablation in these critically ill patients. As a result of numerous VT studies that are currently enrolling patients, some experienced centres needed to make a choice between conflicting studies. This emphasises a future need for a superordinate committee that would collect information on planned trials and combine forces in order to investigate important clinical questions and find an ideal treatment plan for life-threatening arrhythmias.

\section{Potential implications of study results}

In case of a positive result of the study showing that early catheter ablation prior to the first shock reduces the primary endpoint, one could conclude that patients with secondary prevention indication should undergo catheter ablation before ICD implantation. The monitoring data may help to identify parameters that predict heart failure worsening versus occurrence of arrhythmic events in individual patients. In case of a negative study result, 


\section{Table 3 Study organisation}

\begin{tabular}{|c|c|}
\hline Function & Member(s) \\
\hline $\mathrm{CCl}$ & Prof. Karl-Heinz Kuck \\
\hline $\begin{array}{l}\text { Chairman of the steering } \\
\text { committee }\end{array}$ & Prof. Stephan Willems \\
\hline Steering committee & $\begin{array}{l}\text { Prof. Karl-Heinz Kuck } \\
\text { Prof. Stephan Willems } \\
\text { Prof. Stefan Kääb } \\
\text { Prof. Roland Richard Tilz } \\
\text { Prof. Daniel Steven } \\
\text { Prof. Karl Wegscheider } \\
\text { Dr. Klaus Schichı* } \\
\text { Dr. Tino Hauser* }\end{array}$ \\
\hline $\begin{array}{l}\text { Publication steering } \\
\text { committee }\end{array}$ & $\begin{array}{l}\text { Prof. Stephan Willems } \\
\text { Prof. Roland Richard Tilz } \\
\text { Prof. Daniel Steven } \\
\text { Dr. Beate Wenzel } \\
\text { Dr. Annette Thiem* }^{\star}\end{array}$ \\
\hline Data safety monitoring board & $\begin{array}{l}\text { Prof. Günter Breithardt } \\
\text { Prof. Gerhard Steinbeck } \\
\text { Prof. Walter Lehmacher }\end{array}$ \\
\hline $\begin{array}{l}\text { Endpoint assessment } \\
\text { committee and central shock } \\
\text { adjudication board }\end{array}$ & $\begin{array}{l}\text { Prof. Michael Oeff } \\
\text { Prof. Carsten Israel } \\
\text { Prof. Gunnar Klein }\end{array}$ \\
\hline Sponsor & Biotronik SE \& Co. KG \\
\hline Funding provided by & Biotronik SE \& Co. KG \\
\hline ClinicalTrials.gov identifier & NCT02501005 \\
\hline
\end{tabular}

${ }^{*}$ Representatives of Biotronik SE \& Co. KG.

$\mathrm{CCl}$, coordinating clinical investigator.

one could argue that catheter ablation is sufficient after VT recurrence secondary to ICD implantation. Catheter ablation of patients that may not experience future VT episodes may be prevented and patient selection therefore improved.

\section{Study organisation and status}

BERLIN VT study is a trial sponsored by Biotronik SE \& Co. KG, Germany. The steering committee represents the trial leadership. Its members (table 3) are responsible for the scientific and clinical aspects of the trial execution, including reporting of the trial results, and for the reviewing of recommendations by the Data Safety Monitoring Board. The safety board regularly monitors the recruitment and conduct of the trial, timeliness, data quality, the distribution of therapies within the trial groups, serious adverse events and further adverse events selected to their discretion during the course of the trial.

An endpoint assessment committee consisting of three independent clinical experts will review and evaluate all potentially endpoint-related adverse events occurring during the trial. The committee will provide standardised, systematic and unbiased judgement of relevant events for the fulfilment of specified endpoint criteria. The endpoint committee will act also as the central shock adjudication board and will adjudicate all applied ICD shocks for appropriateness, to retrospectively determine protocol compliance in the control group with regard to the timing of ablation procedures.

BERLIN VT started enrollment in July 2015. Until end of January 2018, 134 out of 208 patients have been enrolled. Completion of the enrollment is expected by mid of 2019.

\section{Author affiliations}

${ }^{1}$ University Heart Center Lübeck, Medical Clinic II (Cardiology/Angiology/Intensive Care Medicine), Lübeck, Germany

${ }^{2}$ Department of Cardiology, Asklepios Klinik St. Georg, Hamburg, Germany

${ }^{3}$ Ludwig Maximilians University, Munich, Germany

${ }^{4}$ Institute of Medical Biometry and Epidemiology, University Medical Centre Hamburg-Eppendorf, Hamburg, Germany

${ }^{5}$ Center for Clinical Research, BIOTRONIK SE \& Co. KG, Berlin, Germany

${ }^{6}$ University Heart Center Hamburg-Eppendorf, Hamburg, Germany

${ }^{7}$ Department of Electrophysiology, Heart Center, Köln, Germany

Acknowledgements The authors are thankful to Günther Breithardt and Gerhard Steinbeck for their scientific input, Dörte Vossmeyer for project management and study coordination and Dejan Danilovic for editing of the manuscript.

Contributors KHK, SW, DS and RRT designed the study in collaboration with AT. RRT and DS drafted the manuscript in collaboration with BW, KHK and SW. All authors revised the manuscript critically. All authors have given their final approval of the version to be published.

Funding This study is supported by BIOTRONIK SE \& Co. KG, Berlin, Germany.

Competing interests RRT: Research grants: Abbot, Medtronic, Biotronik, Consultant to Biotronik, Speaker's Bureau Honoraria: Biosense Webster, Medtronic, Abbot, Biotronik, Boston Scientific.

Patient consent for publication Not required.

Provenance and peer review Not commissioned; externally peer reviewed.

Open access This is an open access article distributed in accordance with the Creative Commons Attribution Non Commercial (CC BY-NC 4.0) license, which permits others to distribute, remix, adapt, build upon this work non-commercially, and license their derivative works on different terms, provided the original work is properly cited, appropriate credit is given, any changes made indicated, and the use is non-commercial. See: http://creativecommons.org/licenses/by-nc/4.0/.

\section{REFERENCES}

1. The PCD Investigator Group. Clinical outcome of patients with malignant ventricular tachyarrhythmias and a multiprogrammable implantable cardioverter-defibrillator implanted with or without thoracotomy: an international multicenter study. PCD Investigator Group. J Am Coll Cardiol 1994;23:1521-30.

2. Bardy GH, Troutman C, Poole JE, et al. Clinical experience with a tiered-therapy, multiprogrammable antiarrhythmia device. Circulation 1992;85:1689-98.

3. Bardy GH, Lee KL, Mark DB, et al. Amiodarone or an implantable cardioverter-defibrillator for congestive heart failure. $N$ Engl $\mathrm{J}$ Med 2005;352:225-37.

4. Dickstein K, Cohen-Solal A, Filippatos G, et al. Corrigendum to: ESC Guidelines for the diagnosis and treatment of acute and chronic heart failure 2008. The Task Force for the Diagnosis and Treatment of Acute and Chronic Heart Failure 2008 of the European Society of Cardiology. Developed in collaboration with the Heart Failure Association of the ESC (HFA) and endorsed by the European Society of Intensive Care Medicine (ESICM) [Eur Heart J 2008;29:2388-2442] Eur Heart J 2010;31:624-87.

5. Priori SG, Blomström-Lundqvist C, Mazzanti A, et al. 2015 ESC Guidelines for the management of patients with ventricular arrhythmias and the prevention of sudden cardiac death: The Task Force for the Management of Patients with Ventricular Arrhythmias and the Prevention of Sudden Cardiac Death of the European Society of Cardiology (ESC). Endorsed by: Association for European Paediatric and Congenital Cardiology (AEPC). Eur Heart $J$ 2015;36:2793-867. 
6. Poole JE, Johnson GW, Hellkamp AS, et al. Prognostic importance of defibrillator shocks in patients with heart failure. $N$ Engl J Med 2008;359:1009-17.

7. Ruwald AC, Schuger C, Moss AJ, et al. Mortality reduction in relation to implantable cardioverter defibrillator programming in the Multicenter Automatic Defibrillator Implantation Trial-Reduce Inappropriate Therapy (MADIT-RIT). Circ Arrhythm Electrophysiol 2014;7:785-92.

8. Sood N, Ruwald AC, Solomon S, et al. Association between myocardial substrate, implantable cardioverter defibrillator shocks and mortality in MADIT-CRT. Eur Heart J 2014;35:106-15.

9. de Bakker JM, van Capelle FJ, Janse MJ, et al. Reentry as a cause of ventricular tachycardia in patients with chronic ischemic heart disease: electrophysiologic and anatomic correlation. Circulation 1988;77:589-606.

10. Pogwizd SM, Hoyt RH, Saffitz JE, et al. Reentrant and focal mechanisms underlying ventricular tachycardia in the human heart. Circulation 1992;86:1872-87.

11. Cardiac Arrhythmia Suppression Trial (CAST) Investigators. Preliminary report: effect of encainide and flecainide on mortality in a randomized trial of arrhythmia suppression after myocardial infarction. N Engl J Med 1989;321:406-12.

12. Horowitz LN, Harken AH, Kastor JA, et al. Ventricular resection guided by epicardial and endocardial mapping for treatment of recurrent ventricular tachycardia. N Engl J Med 1980;302:589-93.

13. Wittig JH, Boineau JP. Surgical treatment of ventricular arrhythmias using epicardial, transmural, and endocardial mapping. Ann Thorac Surg 1975;20:117-26.

14. Kuck KH, Cappato R, Siebels J, et al. Randomized comparison of antiarrhythmic drug therapy with implantable defibrillators in patients resuscitated from cardiac arrest : the Cardiac Arrest Study Hamburg (CASH). Circulation 2000;102:748-54.

15. Tilz RR, Lin T, Eckardt L, et al. Ablation Outcomes and Predictors of Mortality Following Catheter Ablation for Ventricular Tachycardia: Data From the German Multicenter Ablation Registry. J Am Heart Assoc 2018;7.

16. Di Biase L, Santangeli P, Burkhardt DJ, et al. Endo-epicardial homogenization of the scar versus limited substrate ablation for the treatment of electrical storms in patients with ischemic cardiomyopathy. J Am Coll Cardiol 2012;60:132-41.

17. Jaïs $\mathrm{P}$, Maury $\mathrm{P}$, Khairy $\mathrm{P}$, et al. Elimination of local abnormal ventricular activities: a new end point for substrate modification in patients with scar-related ventricular tachycardia. Circulation 2012;125:2184-96

18. Marchlinski FE, Callans DJ, Gottlieb CD, et al. Linear ablation lesions for control of unmappable ventricular tachycardia in patients with ischemic and nonischemic cardiomyopathy. Circulation 2000;101:1288-96.

19. Tilz RR, Makimoto H, Lin T, et al. Electrical isolation of a substrate after myocardial infarction: a novel ablation strategy for unmappable ventricular tachycardias--feasibility and clinical outcome. Europace 2014;16:1040-52.

20. Vergara P, Trevisi N, Ricco A, et al. Late potentials abolition as an additional technique for reduction of arrhythmia recurrence in scar related ventricular tachycardia ablation. $J$ Cardiovasc Electrophysiol 2012;23:621-7.

21. Stevenson WG, Khan $H$, Sager $P$, et al. Identification of reentry circuit sites during catheter mapping and radiofrequency ablation of ventricular tachycardia late after myocardial infarction. Circulation 1993;88:1647-70.

22. Stevenson WG, Sager PT, Natterson PD, et al. Relation of pace mapping QRS configuration and conduction delay to ventricular tachycardia reentry circuits in human infarct scars. J Am Coll Cardiol 1995;26:481-8
23. Stevenson WG, Friedman PL, Sager PT, et al. Exploring postinfarction reentrant ventricular tachycardia with entrainment mapping. J Am Coll Cardiol 1997;29:1180-9.

24. Reddy VY, Reynolds MR, Neuzil P, et al. Prophylactic catheter ablation for the prevention of defibrillator therapy. N Engl J Med 2007;357:2657-65.

25. Dinov B, Arya A, Bertagnolli L, et al. Early referral for ablation of scar-related ventricular tachycardia is associated with improved acute and long-term outcomes: results from the Heart Center of Leipzig ventricular tachycardia registry. Circ Arrhythm Electrophysiol 2014;7:1144-51.

26. Frankel DS, Mountantonakis SE, Robinson MR, et al. Ventricular tachycardia ablation remains treatment of last resort in structural heart disease: argument for earlier intervention. $J$ Cardiovasc Electrophysiol 2011:22:1123-8.

27. Rothman SA, Hsia HH, Cossú SF, et al. Radiofrequency catheter ablation of postinfarction ventricular tachycardia: long-term success and the significance of inducible nonclinical arrhythmias. Circulation 1997;96:3499-508.

28. Nakahara S, Tung R, Ramirez RJ, et al. Characterization of the arrhythmogenic substrate in ischemic and nonischemic cardiomyopathy implications for catheter ablation of hemodynamically unstable ventricular tachycardia. J Am Coll Cardiol 2010;55:2355-65.

29. Moss AJ, Schuger C, Beck CA, et al. Reduction in inappropriate therapy and mortality through ICD programming. N Engl J Med 2012;367:2275-83.

30. Gasparini M, Proclemer A, Klersy C, et al. Effect of long-detection interval vs standard-detection interval for implantable cardioverterdefibrillators on antitachycardia pacing and shock delivery: the ADVANCE III randomized clinical trial. JAMA 2013;309:1903-11.

31. Antiarrhythmics versus Implantable Defibrillators (AVID) Investigators. A Comparison of Antiarrhythmic-Drug Therapy with Implantable Defibrillators in Patients Resuscitated from Near-Fatal Ventricular Arrhythmias. N Engl J Med 1997;337:1576-84.

32. Connolly SJ, Gent M, Roberts RS, et al. Canadian implantable defibrillator study (CIDS) : a randomized trial of the implantable cardioverter defibrillator against amiodarone. Circulation 2000;101:1297-302.

33. Goldenberg I, Vyas AK, Hall WJ, et al. Risk stratification for primary implantation of a cardioverter-defibrillator in patients with ischemic left ventricular dysfunction. J Am Coll Cardiol 2008;51:288-96.

34. Tan VH, Wilton SB, Kuriachan V, et al. Impact of programming strategies aimed at reducing nonessential implantable cardioverter defibrillator therapies on mortality: a systematic review and metaanalysis. Circ Arrhythm Electrophysiol 2014;7:164-70.

35. Stevenson WG, Wilber DJ, Natale A, et al. Irrigated radiofrequency catheter ablation guided by electroanatomic mapping for recurrent ventricular tachycardia after myocardial infarction: the multicenter thermocool ventricular tachycardia ablation trial. Circulation 2008;118:2773-82.

36. Kuck KH, Schaumann A, Eckardt L, et al. Catheter ablation of stable ventricular tachycardia before defibrillator implantation in patients with coronary heart disease (VTACH): a multicentre randomised controlled trial. Lancet 2010;375:31-40.

37. Kuck K-H, Tilz RR, Deneke T, et al. Impact of Substrate Modification by Catheter Ablation on Implantable Cardioverter-Defibrillator Interventions in Patients With Unstable Ventricular Arrhythmias and Coronary Artery Disease. Circulation 2017;10.

38. Sapp JL, Wells GA, Parkash R, et al. Ventricular Tachycardia Ablation versus Escalation of Antiarrhythmic Drugs. N Engl J Med 2016;375:111-21.

39. Reddy VY, Neuzil P, Taborsky M, et al. Short-term results of substrate mapping and radiofrequency ablation of ischemic ventricular tachycardia using a saline-irrigated catheter. J Am Coll Cardiol 2003;41:2228-36. 